\title{
ANÁLISE SOCIODEMOGRÁFICA E CLÍNICA DE INDIVÍDUOS COM DIABETES TIPO 2 E SUA RELAÇÃO COM O AUTOCUIDADO
}

Heloisa Carvalho Torres ${ }^{1}$, Ana Emilia Pace ${ }^{2}$, Mayra Alves Stradioto ${ }^{3}$

\begin{abstract}
RESUMO: O objetivo do estudo foi analisar as características sociodemográficas e a sua relação com os conhecimentos gerais, atitudes em diabetes, autogerenciamento dos cuidados e controle metabólico dos indivíduos com diabetes. É um estudo transversal, com uma amostra de 105 indivíduos com diabetes tipo 2, atendidos em ambulatório de referência. Os dados foram coletados mediante entrevistas e avaliados por questionários específicos, em 2006. Os resultados apresentaram, em sua maioria, pessoas do sexo feminino, na faixa etária acima dos 60 anos e escolaridade abaixo de 4 anos. Os testes de atitudes, autocuidado da doença, níveis de hemoglobina glicada e índice de massa corporal não apresentaram diferenças significativas em relação ao sexo, à idade e nível de escolaridade. Concluiu-se a importância de identificar os fatores sociodemográficos e clínico ao se estabelecer o programa educativo em diabetes direcionado às necessidades do indivíduo, visando aumentar a adesão ao tratamento, controle da doença e retardo/prevenção de suas complicações.
\end{abstract}

PALAVRAS-CHAVE: Educação em saúde; Autocuidado; Diabetes mellitus.

\section{SOCIODEMOGRAPHIC AND CLINICALANALYSIS OF INDIVIDUALS WITH TYPE 2 DIABETES AND ITS RELATION TO SELF-CARE}

\begin{abstract}
The aim of this study was to analyze the diabetic patients' demographic characteristics and their relationship with their knowledge and attitudes toward diabetes, self-management of care and metabolic control. It is a cross-sectional study with a sample of 105 individuals with type 2 diabetes treated at a reference clinic. Data were collected through interviews and evaluated by specific questionnaires, in 2006. Results showed mostly females, aged over 60 years, and with less than 4 years of schooling. The tests of attitudes, self-care for the disease, HbA1c and BMI did not differ significantly in relation to gender, age and educational level. In conclusion, the importance of identifying the socio demographic and clinical when establishing a diabetes education program focused on individual needs was stressed, in order to increase adherence to treatment, disease control and delay / prevention of complications.
\end{abstract}

KEYWORDS: Health education; Self care; Diabetes mellitus.

\section{ANÁLISIS SOCIODEMOGRÁFICO Y CLÍNICO DE INDIVIDUOS CON DIABETES TIPO 2 Y SU RELACIÓN CONEL AUTOCUIDADO}

RESUMEN: El objetivo del estudio fue analizar las características socio-demográficas y su relación con los conocimientos generales, actitudes en diabetes, auto-gerenciamiento de los cuidados y control metabólico de los individuos con diabetes. Se trata de un estudio transversal, con una muestra de 105 individuos con diabetes tipo 2, atendidos en ambulatorio de referencia. Los datos fueron recolectados a través de entrevistas y evaluados por cuestionarios específicos, en 2006. Los resultados presentaron, en su mayoría, personas de sexo femenino, en la faja etaria encima de 60 años y de escolaridad debajo de 4 años. Las pruebas de actitudes, auto-cuidado de la enfermedad, niveles de hemoglobina glicosilada e índice de masa corporal no presentaron diferencias significativas en relación al sexo, edad y nivel de escolaridad. Se concluye la importancia de identificar los factores socio-demográficos y clínico al establecerse el programa educativo en diabetes direccionado a las necesidades del individuo, visando aumentar la adherencia al tratamiento, el control de la enfermedad y retraso/prevención de sus complicaciones.

PALABRAS CLAVE: Educación en salud; Autocuidado; Diabetes mellitus.

${ }^{1}$ Enfermeira. Doutora. Professor Adjunto do Departamento de Enfermagem Aplicada da Escola de Enfermagem da Universidade Federal de Minas Gerais-UFMG.

${ }^{2}$ Enfermeira. Doutora. Professor Adjunto da Escola de Enfermagem de Ribeirão Preto-EERP, Departamento de Enfermagem Saúde do Adulto.

${ }^{3}$ Professora. Mestre em Estatística. Professor do Departamento de Matemática e Estatística da Pontificia Universidade Católica de Minas Gerais-PUCMG.

Autor correspondente:

Heloisa de Carvalho Torres

Universidade Federal de Minas Gerais

Av. Alfredo Balena, 190 - 30130-100 - Belo Horizonte-MG

Recebido: 09/03/09

E-mail: heloisa@enf.ufmg.br

Aprovado: 14/10/09

Cogitare Enferm 2010 Jan/Mar; 15(1):48-54 


\section{INTRODUÇÃO}

O Diabetes Mellitus (DM), configura-se como um dos principais problemas de saúde pública, sendo um dos transtornos crônicos mais frequentes do mundo. Das formas existentes, o diabetes tipo 2 responsabiliza-se por, aproximadamente, 90 a $95 \%$ dos casos de diabetes mellitus ${ }^{(1)}$. A urbanização crescente, o estilo de vida pouco saudável, que inclui dieta com alto teor de gordura e diminuição da atividade física, obesidade, e o envelhecimento populacional, são fatores responsáveis pela tendência crescente da incidência da doença na população, gerando um elevado gasto do poder público para o controle e tratamento de suas complicações ${ }^{(1-4)}$. No Brasil, segundo o Ministério da Saúde, 11\% da população (cerca de 5 milhões de pessoas), acima de 40 anos são portadoras de diabetes tipo 2. Essa elevada incidência da doença representa uma grande perda econômica para o país, pois impõe ao indivíduo a diminuição de sua produtividade no trabalho, aposentadoria precoce, limitando assim as capacidades funcionais ${ }^{(1-2)}$.

Segundo a Organização Mundial de Saúde ${ }^{(3)}$, é necessário que o indivíduo com diabetes adote habilidades de autocuidado direcionadas à adesão ao plano alimentar e à prática de atividades físicas diárias que lhe permitam controlar a doença. Quanto maior o acesso à informação e ao conhecimento sobre sua comorbidade, maior será a capacidade aprendida de realizar uma ação de maneira competente, instituindo medidas que lhe permitam a adoção de uma nutrição saudável e a modificação do estilo de vida que irá refletir diretamente na melhoria de sua qualidade de vida ${ }^{(4-6)}$.

Os programas educativos em diabetes, adequados ao contexto sociocultural e econômico dos indivíduos, favorecem uma melhor compreensão da educação em saúde para o autocuidado em diabetes e proporcionam ao indivíduo conhecimentos, habilidades, atitudes e motivação para controlar a doença. A educação para o autocuidado é uma das estratégias mais adequadas e indicadas para o tratamento dos indivíduos, proporcionando alcançar níveis normais ou quase normais de glicose sangüínea ${ }^{(7)}$. Estudos mostram que o controle e a prevenção de complicações do diabetes são possíveis por meio de programas educativos para orientar as habilidades de autocuidado ${ }^{(8-10)}$.

Autores têm procurado identificar quais são os fatores que influenciam o comportamento saudável do indivíduo frente à doença. Os achados dos estudos apontaram que o gênero, idade e nível de escolaridade têm influência na adesão do indivíduo ao autocuidado; as mulheres acima de 60 anos são as que mais aderem à educação do autocuidado. Observa-se relação entre os níveis de escolaridade, sexo, idade, raça, estado socioeconômico, marital e ocupacional e a capacidade dos indivíduos em melhorar os conhecimentos, acessar as informações para o aucuidado e controlar a doença ${ }^{(7-8)}$. O conhecimento dos aspectos sociodemográficos e clínicos dos indivíduos é fator indispensável para planejar e implantar programas educativos em diabetes, visando promover a educação do autocuidado.

Desta forma, o presente estudo tem o propósito de analisar as características sociodemográficas e clínicas e a sua relação com os conhecimentos gerais, atitudes em diabetes, autogerenciamento dos cuidados e controle metabólico avaliado pela hemoglobina glicada (HbA1c) dos indivíduos com DM tipo 2, atendidos no ambulatório de hospital de referência de Belo Horizonte, Minas Gerais.

\section{METODOLOGIA}

O estudo foi planejado sob a forma de um desenho observacional de caráter exploratório descritivo, do tipo transversal, que é utilizado frequentemente para descrever as características da saúde humana ${ }^{(11)}$. A investigação foi desenvolvida com a participação de 105 indivíduos com diabetes tipo 2 de ambos os sexos, com idade entre 30 e 70 anos, em seguimento ambulatorial em hospital de referência de Belo Horizonte. Os sujeitos foram recrutados por meio de registros em prontuários, no período de maio a junho de 2006.

Os critérios de inclusão foram: grau de escolaridade acima da quarta série do ensino fundamental e possibilidade de comparecer bimestralmente, por três vezes. Não foram incluídos indivíduos com incapacidade de leitura, complicações crônicas definidas como insuficiência renal, cegueira, amputação de membros, entre outras.

A coleta de dados foi realizada por um instrumento semiestruturado constituído de questões fechadas. As variáveis contemplaram as dimensões demográficas: idade, sexo e escolaridade e as variáveis de interesse incluíram o conhecimento sobre o diabetes, atitudes (respostas emocionais sobre a doença) e autogerenciamento (adesão à dieta e atividade física). Para tal, foram utilizados, respectivamente, os instrumentos: conhecimento geral 
da doença (DKN-A), atitudes psicológicas (ATT-19) e autogerenciamento dos cuidados do diabetes $(E S M)^{(11)}$, previamente adaptados para o português do Brasil e validados ${ }^{(11)}$. Tais instrumentos foram constituídos, respectivamente, por 15, 19 e 8 questões fechadas, autopreenchíveis e anônimas, aplicados individualmente, com apoio de pessoal treinado.

O teste de conhecimentos DKN-A inclui um escore total de 14 pontos, abordando os conhecimentos sobre o gerenciamento da doença. $\mathrm{O}$ paciente precisa adquirir, no mínimo, um escore de 8 pontos para mostrar uma melhora dos conhecimentos sobre a doença. $\mathrm{O}$ ATT-19 contém 19 itens que descrevem as respostas emocionais sobre a doença, com questões fechadas. Cada enunciado é respondido com a ajuda de uma escala de tipo Likert de cinco pontos, partindo de "grande discordância" até "grande concordância", e tem uma taxa de contagem de 19 a 95 pontos para alcançar uma atitude positiva acerca da doença. Nesta escala o paciente necessita alcançar um escore mínimo de 70 pontos de contagem do instrumento. O teste ESM tem o escore total de 8 pontos. Para mostrar que o paciente alcançou uma mudança de comportamento, deve obter um escore mínimo de cinco pontos.

A coleta de dados foi realizada no ambulatório no período vespertino. Contou com a participação de 8 alunos bolsistas de Enfermagem que preencheram o instrumento dos dados sociodemográficos e auxiliaram os indivíduos com diabetes nos questionários descritos anteriormente, para facilitar o melhor entendimento e permitir a participação das pessoas. Cada indivíduo gastou, em média, de 20 a 30 minutos para completar cada questionário, perfazendo um total de duas horas.

Os indicadores clínicos do controle metabólico foram: exame de hemoglobina glicada (HbA1c), que reflete a média do nível de glicose no sangue durante um período de três meses ${ }^{(1)}$, e as medidas antropométricas (peso, altura e IMC). O exame de HbA1c foi realizado no laboratório do hospital de referência. $\mathrm{O}$ peso e altura foram aferidos em uma balança digital (Modelo PL150, Filizzola Ltda, Brasil) do ambulatório.

Para a avaliação dos resultados da HbA1c e do IMC dos pacientes com diabetes tipo 2, utilizamos os parâmetros preconizados pela Associação Latino Americana de Diabetes ${ }^{(1)}$ Os valores normais desses parâmetros são: HbA1c entre 3,5\% e 7,5\%, e IMC $£ 27 \mathrm{~kg} / \mathrm{m}^{2}$ em homens e $£ 26 \mathrm{~kg} / \mathrm{m}^{2}$ em mulheres.

As análises estatísticas dos dados foram realizadas nos programas SPSS V.16 e Minitab, processados com dupla digitação dos dados para controle dos possíveis erros. A caracterização da amostra foi feita pelo teste $t$-Student, tendo sido confirmada a suposição de normalidade dos dados por meio do teste de Kolmogorov-Smirnov. No caso das comparações entre proporções, foi utilizado o teste qui-quadrado $\left(\mathrm{c}^{2}\right)$. Para todas as análises, foi utilizado um nível de significância de 5\%. Destaca-se que para a variável 'idade' foram consideradas duas categorias: menor de 50 anos, e igual ou maior de 50 anos, sendo a idade mínima de 30 anos e a máxima de 79 .

O projeto foi aprovado pelo Comitê de Ética em Pesquisa da Universidade Federal de Minas Gerais, parecer $\mathrm{n} \stackrel{0}{157 / 06}$, e todos os participantes assinaram o Termo de Consentimento Livre e Esclarecido no momento da consulta de enfermagem.

\section{RESULTADOS}

A distribuição dos participantes com relação às variáveis sociodemográficas (idade, sexo e grau de escolaridade) encontram-se na tabela 1. Foram estudados 105 indivíduos diabéticos entre 30 e 79 anos. Destes, 26 (24,8\%) eram do sexo masculino e 79 (75,2\%) do feminino, não havendo diferença estatisticamente significante entre os sexos com relação à idade $(\mathrm{p}=0,363)$ e escolaridade $(\mathrm{p}=0,804)$. A grande maioria tinha 50 anos ou mais $(84,8 \%)$ e até $1^{\circ}$ grau completo $(79,0 \%)$.

Tabela 1 - Distribuição percentual da amostra de indivíduos com diabetes tipo 2. Belo Horizonte, 2006.

\begin{tabular}{lll}
\hline Variáveis sociodemográficas & $\mathrm{n}$ & $\%$ \\
\hline
\end{tabular}

\section{Sexo}

Feminino

Masculino

\section{Faixa etária}

$<$ de 50 anos

50 a 59 anos

60 anos ou mais

56

53,3

Escolaridade (anos de estudo)

Até 4 anos

5 a 8 anos

$31 \quad 30,1$

Mais de 8 anos

$22 \quad 21,4$


As tabelas que seguem (2,3, 4 e 5) demonstram os resultados em média e desvio padrão da relação estabelecida e estudada entre as variáveis sóciodemográficas (sexo, idade e escolaridade) e as clínicas (hemoglobina glicada, peso, altura, IMC) associados aos escores dos instrumentos do autogerenciamento dos cuidados, atitudes psicológicas e conhecimentos sobre a doença.

Na Tabela 2, observamos que não houve diferença estatisticamente significante entre os indivíduos do sexo masculino e feminino quanto ao valor da hemoglobina glicada ( $\mathrm{p}=0,925)$, nem em relação ao peso $(p=0,734)$ e ao IMC $(p=0,220)$. Os indivíduos do sexo masculino apresentaram altura estatisticamente maior do que indivíduos do sexo feminino $(p=0,042)$.

Homens e mulheres também não diferiram estatisticamente com relação à pontuação nos questionários de autogerenciamento dos cuidados, atitudes e conhecimentos.

Tabela 2 - Média e desvio-padrão das variáveis em relação ao sexo dos indivíduos com diabetes tipo 2 do ambulatório do hospital de referência de Belo Horizonte, 2006.

\begin{tabular}{lcccc}
\hline Variáveis & $\begin{array}{c}\text { Masculino } \\
(\mathrm{n}=26)\end{array}$ & $\begin{array}{c}\text { Feminino } \\
(\mathrm{n}=79)\end{array}$ & $\mathrm{t}$ & $\mathrm{p}$ \\
\hline Hemoglobina glicada & $9,55 \pm 2,49$ & $9,50 \pm 2,44$ & $-0,095$ & 0,925 \\
Peso $(\mathrm{kg})$ & $74,52 \pm 16,60$ & $75,66 \pm 14,05$ & 0,341 & 0,734 \\
Altura $(\mathrm{cm})$ & $1,61 \pm 0,09$ & $1,57 \pm 0,08$ & $-2,062$ & $0,042 *$ \\
IMC & $28,91 \pm 7,22$ & $30,54 \pm 5,30$ & 1,233 & 0,220 \\
Autogerenciamento dos cuidados & $3,80 \pm 0,96$ & $3,55 \pm 0,80$ & $-1,284$ & 0,202 \\
Atitudes psicológicas & $65,58 \pm 10,57$ & $64,08 \pm 10,78$ & $-0,619$ & 0,538 \\
Conhecimentos sobre diabetes & $8,54 \pm 2,82$ & $9,52 \pm 2,53$ & 1,668 & 0,098 \\
\hline D
\end{tabular}

Dados em média \pm desvio-padrão

"Diferença estatisticamente significante

Em relação à idade dos indivíduos, conforme mostrado na tabela 3, nenhuma variável apresentou diferença estatisticamente significante entre os grupos de idades dos indivíduos ( $\mathrm{p}>0,05)$.

Tabela 3 - Média e desvio-padrão das variáveis em relação à idade dos indivíduos com diabetes tipo 2 do ambulatório do hospital de referência de Belo Horizonte, 2006.

\begin{tabular}{lccccc}
\hline & \multicolumn{5}{c}{ Idade } \\
Variáveis & $<$ de 50 anos & 50 a 59 anos & 60 anos ou mais & $\mathrm{t}$ & $\mathrm{p}$ \\
& $(\mathrm{n}=16)$ & $(\mathrm{n}=33)$ & $(\mathrm{n}=56)$ & & \\
\hline Hemoglobina Glicada & $9,27 \pm 2,23$ & $9,92 \pm 2,47$ & $9,33 \pm 2,48$ & 0,684 & 0,507 \\
Peso $(\mathrm{kg})$ & $78,63 \pm 12,75$ & $72,76 \pm 14,88$ & $76,02 \pm 15,09$ & 0,947 & 0,391 \\
Altura $(\mathrm{cm})$ & $1,56 \pm 0,08$ & $1,56 \pm 0,08$ & $1,60 \pm 0,09$ & 2,640 & 0,076 \\
IMC & $32,17 \pm 3,92$ & $29,70 \pm 5,59$ & $29,84 \pm 6,41$ & 1,056 & 0,352 \\
Autogerenciamento dos Cuidados & $3,57 \pm 0,98$ & $3,75 \pm 0,73$ & $3,57 \pm 0,85$ & 0,685 & 0,507 \\
Atitudes & $61,67 \pm 9,28$ & $64,39 \pm 11,27$ & $65,34 \pm 10,81$ & 0,825 & 0,441 \\
Conhecimentos & $9,13 \pm 3,09$ & $9,82 \pm 2,32$ & $9,09 \pm 2,59$ & 1,100 & 0,337 \\
\hline
\end{tabular}

Dados em média \pm desvio-padrão 
A Tabela 4 mostra que os resultados dos entre si quanto ao nível de escolaridade dos indivíduos indicadores clínicos e dos questionários não diferem $(p>0,05)$.

Tabela 4 - Média e desvio-padrão das variáveis em relação ao nível de escolaridade dos indivíduos com diabetes tipo 2 do ambulatório do hospital de referência de Belo Horizonte, 2006.

Nível de escolaridade (anos de estudo)

\begin{tabular}{lccccc} 
Variáveis & Até 4 anos & De 5 a 8 anos & Mais de 8 anos & $t$ & $p$ \\
& $(\mathrm{n}=50)$ & $(\mathrm{n}=31)$ & $(\mathrm{n}=24)$ & & \\
\hline Hemoglobina glicada & $9,48 \pm 2,47$ & $9,64 \pm 2,45$ & $9,15 \pm 2,22$ & 0,272 & 0,762 \\
Peso (kg) & $76,39 \pm 13,83$ & $74,72 \pm 16,90$ & $74,76 \pm 13,72$ & 0,161 & 0,852 \\
Altura (cm) & $1,59 \pm 0,08$ & $1,57 \pm 0,09$ & $1,57 \pm 0,09$ & 0,804 & 0,450 \\
IMC & $30,25 \pm 5,93$ & $30,14 \pm 6,54$ & $30,30 \pm 4,56$ & 0,005 & 0,995 \\
Autogerenciamento dos cuidados & $3,58 \pm 0,85$ & $3,59 \pm 0,82$ & $3,82 \pm 0,84$ & 0,689 & 0,505 \\
Atitudes & $63,48 \pm 11,43$ & $64,58 \pm 10,33$ & $67,27 \pm 9,52$ & 0,955 & 0,388 \\
Conhecimentos sobre a doença & $9,00 \pm 2,54$ & $9,10 \pm 2,18$ & $10,36 \pm 3,06$ & 2,335 & 0,102 \\
\hline
\end{tabular}

Dados em média \pm desvio-padrão

Em relação à tabela 5, não houve diferença estatisticamente significante entre os indivíduos com hemoglobina glicada normal e alterada, quanto ao resultado dos questionários ( $\mathrm{p}>0,05)$.

As análises indicam que não existe correlação estatisticamente significante entre o nível de hemoglobina glicada e os resultados dos questionários de autocuidado da doença, atitude e conhecimento, assim como em relação às variáveis sociodemográficas $(\mathrm{p}>0,05)$.

Tabela 5 - Média e desvio-padrão dos resultados dos questionários em relação à hemoglobina glicada dos indivíduos com diabetes tipo 2 do ambulatório do hospital de referência de Belo Horizonte, 2006.

\begin{tabular}{lllll}
\hline & Hemoglobina glicada & & & \\
Questionários & $\begin{array}{l}\text { Normal - menor ou igual a } \\
7,5 \%\end{array}$ & $\begin{array}{l}\text { Alterada - maior que } \\
7,5 \%\end{array}$ & $\mathrm{t}$ & $\mathrm{p}$ \\
& $(\mathrm{n}=31)$ & $\mathrm{n}=74)$ & & \\
\hline Autogerenciamento dos cuidados & $3,74 \pm 0,79$ & $3,58 \pm 0,85$ & 0,917 & 0,362 \\
Atitudes psicológicas & $64,60 \pm 9,43$ & $64,47 \pm 11,28$ & 0,054 & 0,957 \\
Conhecimentos sobre a doença & $9,07 \pm 2,83$ & $9,43 \pm 2,49$ & $-0,653$ & 0,516 \\
\hline
\end{tabular}

Dados em média \pm desvio-padrão

\section{DISCUSSÃO E IMPLICAÇÕES}

De acordo com os resultados encontrados na tabela 1, pode-se observar maiores frequências de pessoas com mais de 50 anos de idade (84, 7\%), sexo feminino $(75,2 \%)$ e escolaridade acima de 5 anos (71,5\%). Outro aspecto de relevância é o envelhecimento da população, onde os idosos deixaram de representar apenas 5\% (década de 70) para compor, aproximadamente, $9 \%$ do nosso total de habitantes no ano $2000^{(13)}$. Deve-se levar em consideração a relação entre este envelhecimento e associação com a presença de doenças crônicas não transmissíveis, em especial o diabetes, mais prevalentes entre as mulheres e as pessoas de maior idade ${ }^{(14)}$. Estudos nacionais ${ }^{(3)}$ e internacionais ${ }^{(4,6)}$ têm 
demonstrado que o DM tem um pico de incidência ao redor dos 50 anos de idade, com a prevalência de 97\% dos indivíduos. Os resultados apresentados no estudo requerem uma atenção diferenciada dos profissionais de saúde para que os programas educativos em diabetes sejam adequados às características sociodemográficas do indivíduo, com vistas a favorecer a adesão ao tratamento e promover o controle metabólico.

Ao estudar a relação entre as variáveis clínicas, autogerenciamento dos cuidados, atitudes e conhecimentos sobre DM em relação às variáveis sociodemográficas, não houve associação estatisticamente significativa (Tabela 2, 3, 4,5). Estudos apontam que o baixo conhecimento sobre a doença está relacionado com a idade, escolaridade, tempo de tratamento, função cognitiva, sexo e nível de depressão(11), enquanto que outras pesquisas evidenciaram que o conhecimento não estava relacionado com o controle glicêmico ${ }^{(12)}$. É relevante considerar os aspectos psicológicos, sociais e culturais do viver dos indivíduos para que se possa obter uma mudança de comportamento, ocasionando melhor convivência com a doença ${ }^{(15-16)}$.

Os resultados apresentados na tabela 2 apontaram que a média do escore do questionário de conhecimentos em relação à doença foi maior do que esperado, que o "conhecimento" é apenas uma das variáveis que pode modificar as atitudes de aceitação do indivíduo frente ao manejo do diabetes. De forma complementar, a literatura coloca que o indivíduo com diabetes tem que passar por seis fases de adaptação em relação à doença, que vão desde o constrangimento do diagnóstico, a vontade de aprendizagem, o medo, aceitação, a gestão do diabetes até o processo de empoderamento ${ }^{(17)}$. Conhecer estas etapas, bem como as representações do indivíduo sobre o diabetes e suas reações emocionais, é crucial para uma abordagem holística e humanizada.

Em estudos anteriores de longa duração realizados entre indivíduos com diabetes tipo 2, com a finalidade de avaliar o programa educativo por meio das variáveis de conhecimentos e atitudes sobre DM e autocuidado e sua interferência no controle metabólico da doença, identificou-se que a educação é fundamental para o autogerenciamento dos cuidados e auxilia na redução de complicações crônicas ${ }^{(10-11)}$. Nesse sentido, também observou-se que a educação voltada para a prevenção e o controle em diabetes apresenta um desafio quer para os indivíduos, quer para profissionais de saúde, visando alcançar melhorias no autocuidado da doença e na promoção da saúde por tratar-se de uma doença que afeta diferentes faixas etárias, independente do nível de instrução e condições sociais de vida.

Os resultados apresentados nesse estudo podem se constituir de parâmetros na adequação aos programas educativos em saúde, em especial em diabetes, para que haja uma maior compreensão e motivação por parte dessas pessoas. Assim, torna-se essencial a conscientização dos profissionais de saúde frente aos aspectos clínicos e demográficos da população às quais estão dirigidos, de modo a estabelecer uma educação estruturada e programas de intervenção, de maneira contínua e progressiva, para que os indivíduos com diabetes alcancem o controle metabólico e uma vida normal com a doença.

\section{CONCLUSÃO}

Com base nos resultados deste estudo, observou-se que os dados clínicos e demográficos dos indivíduos com DM tipo 2 atendidos no ambulatório do hospital de referência de Belo Horizonte, são de grande valor para estabelecer estratégias educativas direcionadas às necessidades dos indivíduos e definir metas a serem traçadas quanto ao manejo da doença.

Acredita-se que estes achados possam servir como estímulo para o aprofundamento dos estudos relacionados às diferenças demográficas e clínicas de outros pacientes, bem como servir para planejar, organizar e executar um programa educativo em DM. Além disso, espera-se que os profissionais de saúde responsáveis pelas intervenções educativas alcancem resultados desejáveis na educação do indivíduo com diabetes, como um aspecto fundamental do cuidado na obtenção do controle da doença e, assim, prevenir ou retardar o desencadeamento de complicações agudas e crônicas, ajudando-os na promoção da qualidade de vida.

\section{REFERÊNCIAS}

1. Ministério da Saúde(BR). Data SUS. Taxa de prevalência de diabetes mellitus. [Acesso em 2009 Mai 13]. Disponível: http://tabnet.datasus.gov.br/cgi/idb1998/ fqd09.htm.

2. Ministério da Saúde (BR). Secretaria de Atenção à Saúde. Departamento de Atenção Básica. Diabetes Mellitus. Brasília; 2006: 56 p. 
3. Organização Mundial da Saúde. Cuidados inovadores para condições crônicas: componentes estruturais de ação: relatório mundial. Brasília: OMS, 2003. 105 p.

4. Wild S, Roglic G, Green A, Sicree R, King H. Global prevalence of diabetes: estimatives for the year 2000 and projections for 2030. Diabets Care. 2004 May;7(5):104753.

5. Otero LM, Zanetti ML, Teixeira CRS. Sociodemographic and clinical characteristics of a diabetic population at a primary level health care center. Rev Latino-Am Enfermagem. 2007 Set/Out;15:768-73.

6. American Diabetes Association. Diagnosis and classification of diabetes mellitus. Diabetes Care 2007;30(S42-S47):89-94.

7. Torres HC, Franco L, Stradioto M, Hortale V, Shall V. Avaliação estratégica de educação em grupo e individual no programa educativo em diabetes. Rev Saúde Pública 2009;43(2):431-9.

8. Xu Y, Toobert D, Savage C, Pan W, Whitmer K. Factors influencing diabetes self-management in chinese people with type 2 Diabetes. Res Nurs Health. 2008;31(6):613-25.

9. Pace EA, Nunes DP, Vigo OK. O conhecimento dos familiares acerca da problemática do portador de diabetes mellitus. Rev. Latino-Am Enfermagem. 2003;11(2):312-9.

10. Pereira MG. Epidemiologia: teoria e prática. Rio de Janeiro. Guanabara Koogan; 1995

11. Torres HC, Hortale VA, Shall V. A experiência de jogos em grupos operativos na educação em saúde para diabéticos. Cad Saúde Pública. 2003;19(4):1039-47.

12. Pace AE, Vigo KO, Caliri MHL, Fernandes APM. O conhecimento sobre diabetes mellitus no processo de autocuidado. Rev Latino-Am Enfermagem. 2006 Set/Out; 11(3):728-34.

13. Torres HC, Hortale VA, Shall VT. Validação dos questionários de conhecimento (DKN-A) e atitude (ATT-19) de diabetes mellitus. Rev Saúde Pública. 2005; 39(6):906-11.

14. Gimenes HT, Zanetti ML, Haas VJ. Factors related to patient adherence to antidiabetic drug therapy. Rev Latino-Am Enfermagem. 2009 Jan/Fev;17(1):46-51.

15. Grillo MFF, Gorini MIPC. Caracterização de pessoas com diabetes mellitus tipo 2. Rev Bras Enferm. 2007 Jan/Fev;60(1):49-54.

16. Sedrez CKL, Spadari G. Estilo de vida e saúde: condicionantes de um envelhecimento saudável Cogitare Enferm 2008; 13( 2):252-60.

17. Abdoli S, Ashktorab T, Ahmadi F, Parvizi S, Dunnin T. The empowerment process in people with diabetes: an iranian perspective. Int Nurs Rev. 2008;55(4):447-53. 